\title{
La política y lo político en Twitter: Análisis del discurso de los candidatos presidenciales de Colombia
}

\author{
Luis Eduardo Ruano $\mathrm{I}^{1}$, Juan Camilo López $\mathrm{M}^{2}$, y Jonathan Felipe Mosquera $\mathrm{C}^{3}$ \\ luiseruanoi@gmail.com,juanlop@unicauca.edu.co, felipemosquera@unicauca.edu.co \\ 1 Profesor investigador. Universidad Cooperativa de Colombia. Grupo de Investigación Psiedu. Popayán, \\ Cauca, Colombia. \\ 2 Investigador. Grupo de Investigación Psiedu. Popayán, Cauca, Colombia. \\ 3 Investigador. Grupo de Investigación Psiedu. Popayán, Cauca, Colombia.
}

DOI: 10.17013/risti.28.57-71

\begin{abstract}
Resumen: Las nuevas redes sociales virtuales, operan como espacios de debate, fundamentales para entender los procesos electorales contemporáneos. Aunque la efectividad estratégica de las redes no este comprobada, pues dependen de múltiples elementos contextuales, sus características y particularidades, han asumido progresivamente mayor relevancia en el marco de los análisis de las ciencias sociales y del comportamiento, a la vez que se insertan en los discursos y prácticas de candidatos, equipos asesores y partidos políticos. En este trabajo, se analizan discursos de los candidatos a la presidencia de Colombia (2018), que tienen lugar en Twitter, demostrando la manera en que los mismos configuran la construcción de una dimensión política. Tomando como base un estudio netnográfico, se interpreta un corpus documental de tweets, emitidos por cuatro candidatos presidenciales, clasificados a partir de cinco categorías de análisis (Corrupción, educación, salud, desempleo y paz).
\end{abstract}

Palabras clave: Colombia; elecciones; redes sociales virtuales; tipologías; discurso.

\section{Politics and political on Twitter: Analysis of the discourse of the presidential candidates of Colombia}

Abstract: The new virtual social networks operate as spaces for debate, these are fundamental to understanding contemporary electoral processes. Although the strategic effectiveness of the networks is not proven, since they depend on multiple contextual elements, their characteristics and particularities have assumed progressively greater relevance in the context of the social and behavioral sciences analysis, while they are inserted in the speeches and practices of candidates, advisory teams and political parties. In this paper, discourses of candidates for the presidency of Colombia (2018), which take place on Twitter, are analyzed, demonstrating the way in which they shape the construction of a political dimension. Based on a netnographic study, a documentary corpus of tweets is interpreted, issued by 
four presidential candidates, classified from five categories of analysis (Corruption, education, health, unemployment and peace).

Keywords: Colombia; Elections; virtual social networks; Types; Speech.

\section{Introducción}

Las redes sociales virtuales, constituyen nuevas formas de abordar la estructuración de la política, las elecciones y elementos propios de los Estados, como la gobernabilidad y la gobernanza (Mayorga \& Cordova, 2007). Este documento, tiene como objetivo general, analizar la construcción de la dimensión política del discurso de los candidatos presidenciales en Colombia en la red social virtual de Twitter en octubre del año 2017.

Se describen dos momentos de análisis, en el primero, se identifican los perfiles políticos de los cuatro candidatos presidenciales con mayor intención de voto, la elección de los mismos se realiza teniendo en cuenta las encuestas nacionales (Centro Nacional de consultoría, febrero de 2018 y Cifras y conceptos, marzo de 2018) y un ejercicio estadístico desarrollado en noviembre de 2017 por los investigadores de este trabajo y que motivó el análisis cualitativo, que aquí se presenta. Seguidamente se procede al análisis del discurso de los tweets emitidos por los candidatos presidenciales, teniendo en cuenta cinco códigos, considerados como las principales problemáticas a resolver del próximo presidente de Colombia. El corpus sobre el cual se realiza el análisis consta de publicaciones realizadas por los candidatos, en un espacio de tiempo cercano al ejercicio estadístico mencionado. Los códigos, responden a frecuencias con puntuación elevada en la misma encuesta. Entre tanto, debe aclararse que no se postula una investigación de carácter mixto, sino un ejercicio de orden cualitativo, que toma como referencia un previo trabajo de pretensiones cuantitativas.

Es menester mencionar que el presente trabajo de investigación es la continuación y extensión del capítulo de libro titulado "Dimensión política del discurso en los candidatos presidenciales de Colombia: un análisis de la red social twitter” (Ruano, López \& Mosquera, 2018)

\section{Aproximación teórica}

El discurso analizado como hecho social, según lo expuesto conlleva según Durkheim (1997), al establecimiento de variables determinantes e incidentes en la idea política del candidato, expresados en los tweet emitidos, en el contexto de la democracia contemporánea. Su dimensión política implica referir a la conformación de carácter político del discurso, pues como lo expone Mouffe (2011), ignorar esto en la política, es no lograr advertir de las complejidades de la pluralidad, las cuales conllevan a la formación de identidad política.

Echeverría (2011) advierte que la política tiene por referencia concreta a las instituciones, sin embargo, esta no debe ser analizada sin concepciones previas de lo político, pues este elemento se construye en la vida cotidiana de los ciudadanos, a partir de la confrontación entre ideas, evidentemente opuestas entre sí. 
En términos de Retamozo (2010), lo político hace referencia a lo instituyente y la política a lo instituido, advirtiendo de los debates que han existido en torno a la existencia (o no) de conflictos y disputas en el terreno de lo político, pues según Arendt (1997), es solo un espacio de libertad, deliberación, en donde los puntos de vista serán respetados.

La incorporación del discurso, como un elemento para la constitución de hegemonía, en el plano de la disputa de lo político, es señalada por Castoriadis (2007), teniendo como consecuencia de la conformación de imaginarios sociales, lo cual hace referencia a la política. Así mismo, Mouffe (2011), ubica a la política en el campo empírico, mientras que considera lo político propio de las discusiones en la teoría política.

Estas perspectivas conceptuales, permiten establecer criterios comunes y discrepancias en algunos autores, sin embargo, es de destacar que la presente investigación ubica dichos elementos en el ámbito de las redes sociales virtuales, generando nuevos retos de análisis en las Ciencias Sociales.

El elemento de lo político, será entendido con base en la relación conflictiva generada a partir del amigo/enemigo, precedida por un nosotros/ellos, lo cual será empíricamente observable en la biografía de los candidatos presidenciales y de manera más específica en los tweets que estos emiten, logrando generar identidad política. Este debate, conlleva a las respuestas favorables (o no) por parte de las audiencias, pues el fin último es atraer mayor número de votantes. Esto, en última instancia (la política), dará como consecuencia resultados electorales mediado (entre otras cosas) por las interacciones generadas a partir del discurso.

De este modo, en la red social virtual de Twitter, lo político es entendido a partir de las posiciones discursivas de los candidatos presidenciales, siendo esto un hecho social, que surge en su pasado y se da a través de las interacciones de los mismos en la esfera pública. A su vez, la política se plantea como el resultado de estas posiciones discursivas mediante las reacciones de los usuarios de Twitter y finalmente en los resultados electorales.

\section{Metodología}

La investigación se inscribe en el enfoque cualitativo (Strauss \& Corbin, 1998). Entre tanto, es pertinente aclarar que la idea de investigación surge de la realización de un sondeo de orden cuantitativo, realizado por los investigadores en noviembre del año 2017, el cual pretendía identificar perspectivas electorales, nociones de paz y territorio en los ciudadanos de la ciudad de Popayán.

El sondeo fue realizado de manera presencial con la colaboración de los estudiantes del programa de psicología de la Universidad Cooperativa de Colombia, permitiendo mayor veracidad de la información suministrada. Se estableció un método de muestreo aleatorio sistemático (Ancona, 2004), abordando las nueve comunas de la ciudad. La población universo estuvo compuesta por hombres y mujeres mayores de 18 años (población en edad de votar), residentes en la zona urbana de la ciudad de Popayán. Para la selección de la muestra, fue aplicada la siguiente formula: 


$$
n=\frac{\mathrm{Z} \wedge 2^{*} \mathrm{P}^{*} \mathrm{Q}}{\mathrm{E} \wedge 2^{*} \mathrm{~N}+\mathrm{Z} \wedge 2^{*} \mathrm{P}^{*} \mathrm{Q}}{ }^{1}
$$

Una vez realizada la encuesta, que contó con una muestra de 387 informantes, se procedió a la sistematización de datos en Excel para su respectiva codificación (Sant, Ramos, Pocrifka, \& Simonian, 2016) y depuración de datos. Los resultados de la encuesta fueron generados mediante el software de SPSS, los cuales coligieron en la necesidad de una investigación de enfoque cualitativo, que explicará los resultados de uno de los reactivos de la encuesta: para usted, ¿̇cuál es el principal problema a resolver del próximo presidente de Colombia?

Se utilizó la técnica de investigación netnográfica (Turpo, 2008), a través de la red social virtual de Twitter, frecuentemente recurrida por los candidatos presidenciales para expresar sus ideas programáticas y obtener mayor número de votantes. De este modo, se recolectaron todos los tweets del mes de octubre (mes anterior a la realización de la encuesta) expuestos por los cuatro candidatos presidenciales con mayor intención de voto: Gustavo Petro (GP), Sergio Fajardo (SF), German Vargas (GV), Iván Duque (ID). El corpus se compuso por un total de 215 twets, los cuales fueron examinados a la luz de la propuesta de análisis sociológico del sistema de discursos, según lo planteado por Fernando Conde Gutiérrez (2009). Dicho análisis se planteó en 4 niveles:

En el primer nivel, se procedió a la recolección de los tweets publicados (1 octubre- 31 octubre), relacionados con las cinco temáticas que según las personas encuestadas en la ciudad de Popayán, son el principal problema a resolver del próximo presidente en Colombia (Educación, salud, paz, corrupción, desempleo), generando así códigos deductivos. Se realizó recolección de todos los tweets, pues si bien algunos no poseen la palabra específica de las principales problemáticas a resolver, existen elementos latentes, los cuales permiten establecer una relación del tweet con el tema (Ruano, Congote \& Torres, 2016).

El segundo nivel, se efectuó a partir de micro análisis de los discursos, expuestos por los candidatos presidenciales en los tweets seleccionados. Para esto se dio configuración a 20 códigos abiertos de carácter inductivo (Strauss \& Corbin, 1998), sistematizados y

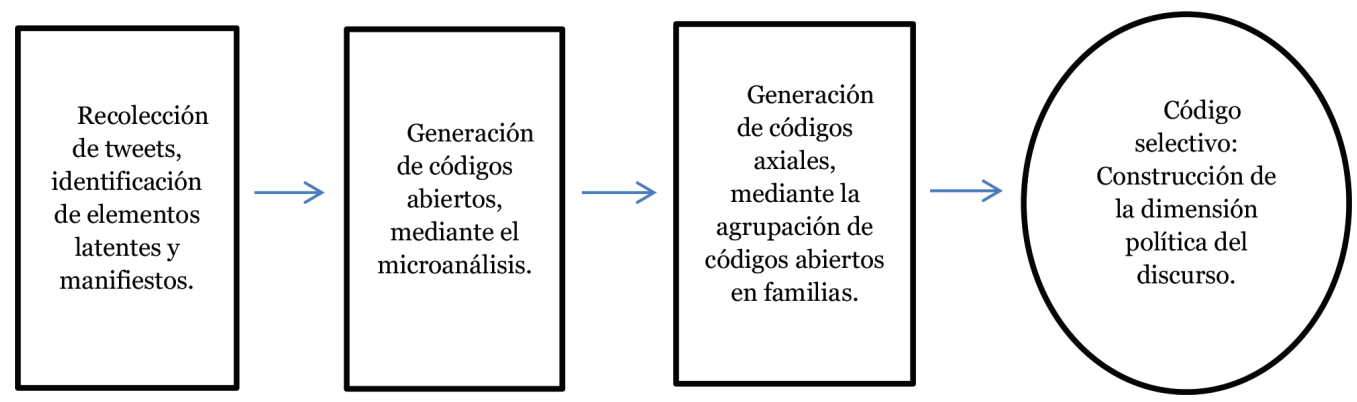

Figura No 1 - Metodología de la investigación

\footnotetext{
${ }^{1} \mathrm{Z}$ : Nivel de confianza, el cual es igual a 95\%; p: Variabilidad positiva, el cual es igual a o,5; q: Variabilidad negativa, la cual es igual a 0,$5 ; \mathrm{N}$ : Población objetivo, la cual es igual a 175.325 personas; E: Error relativo, el cual es igual a 0,005.
} 
procesados mediante el software Atlas.ti. El tercer nivel, se encargó de la construcción de familias en el software Atlas.ti, para así constituir códigos axiales (Strauss \& Corbin, 1998). En última instancia, se agruparon los códigos abiertos, axiales y la dimensión política de los candidatos (partidos políticos, ideología), para así configurar un único código selectivo (Strauss \& Corbin, 1998). La figura No 1, sintetiza el proceso metodológico:

\subsection{Construcción de códigos}

Teniendo como referencia los códigos deductivos, producto de la encuesta, se procedió a la identificación de códigos inductivos a partir del análisis línea a línea (Strauss \& Corbin, 1998) de los tweets. De este modo, la clasificación inicial de cada uno de los tweets se registró mediante veinticinco códigos

El código deductivo denominado “desempleo”, generó cinco códigos inductivos explicados a continuación:

- Informalidad: Tweets que expresan una preocupación del candidato por aquellas personas que trabajan sin las condiciones legales y sociales necesarias.

- Ocupación: Tweets que expresan preocupación por las personas sin trabajos estables y concretos.

- Prensa: Tweets que expresan preocupación por ocultar los verdaderos indicadores de desempleo, por parte de los medios de comunicación.

- Privatización: Tweets que expresan soluciones, alternativas y/o críticas al rol de las empresas privadas en los indicadores de desempleo.

- Sindicatos: Tweets que recalcan la importancia sindicales, teniendo gran incidencia en la generación o no de empleos.

El código deductivo denominado "salud", genera la configuración de cuatro códigos inductivos explicados a continuación:

- Afiliados: Tweets que expresan preocupación por el número o modo de afiliación de las personas al sistema de salud Colombiano.

- Calidad: Tweets que expresan la necesidad de mejorar la atención, garantías e infraestructura en el sistema de salud colombiano.

- Eficiencia/eficacia: Tweets que expresan la preocupación por cumplir metas. Hacer más con menos constituye otro de los temas de preocupación.

- Politización: Tweets, que expresan preocupación por políticos y el modo en que estos afectan al sistema de salud.

- Recursos: Tweets que expresan alternativas de solución al sistema de salud, mediante el aumento del presupuesto nacional.

El código deductivo denominado “educación”, genera la configuración de cuatro códigos inductivos explicados a continuación:

- Calidad: Tweets que expresan preocupación por el funcionamiento de las instituciones educativas públicas de Colombia, proponiendo así, mejoras de infraestructura, alimentación y horarios.

- Gratuidad: Tweets que expresan opiniones de la educación primaria, secundaria y/o superior sin ningún tipo de costo o restricciones. 
- Maestros: Tweets que se preocupan por la generación de propuestas en torno a los docentes de la educación pública en Colombia.

- Presupuesto: Tweets que propongan alternativas de solución a los problemas de la educación pública en Colombia, mediante una mayor inversión.

- Sociales: Tweets que expresan necesidades educativas desde la sociedad.

- Universalidad: Tweets que expresan preocupación por la inclusión en la educación.

El código deductivo denominado "paz", genera la configuración de cinco códigos inductivos explicados a continuación:

- Amnistía e impunidad: Tweets que demuestran preocupación por el perdón e indulto en el proceso de paz para miembros de las FARC-EP y el ELN.

- Cultivos de uso ilícito: Tweets que expresan preocupación por el aumento de la siembra de cultivos de uso ilícito.

- Institucional: Tweets que expresan la necesidad de construir paz a partir de las instituciones.

- Líderes sociales: Tweets que expresan preocupación por el aumento constante de amenazas y/o homicidios a líderes sociales en diferentes territorios de Colombia.

- Seguridad: Tweets que expresan la necesidad de seguridad, en el sector rural y urbano de Colombia.

El código deductivo denominado “corrupción”, genera la configuración de cuatro códigos inductivos explicados a continuación:

- Social: Tweets que manifiestan el inicio de la corrupción desde aspectos sociales como la familia.

- Lucha contra corrupción: Tweets que invitan a la ciudadanía a votar con el fin de derrotar la corrupción.

- Institucional: Tweets que afirman que deben establecerse medidas para luchar contra la corrupción en las instituciones, principalmente las Estatales.

- Ética: Tweets que alegan que la ética o temas de buen gobierno se hacen clave para la lucha contra la corrupción.

La tabla No 1 sintetiza la organización de códigos.

\section{Dimensión política del discurso}

Para el análisis de los discursos, presentes en la red social virtual Twitter de los candidatos presidenciales en Colombia, se analizará el perfil biográfico de cada candidato, brindado por cada una de las páginas de campaña presidenciales de los mismos. Posteriormente se relacionarán los tweets emitidos con los códigos axiales y abiertos, para su respectivo microanálisis.

\subsection{Perfil biográfico de los candidatos}

Gustavo Petro (GP), fue militante del grupo armado M-19, tuvo gran incidencia en el proceso de desarme de esta guerrilla. Candidato de coalición de partidos de izquierda, senador de la república en diferentes periodos y ex alcalde de Bogotá (La Silla Vacía, 2016). 


\begin{tabular}{|c|c|c|}
\hline Códigos abiertos & Códigos axiales & Código selectivo \\
\hline Informalidad & \multirow{5}{*}{ Desempleo } & \multirow{25}{*}{$\begin{array}{l}\text { Construcción de la dimensión política de los } \\
\text { discursos de los candidatos presidenciales en } \\
\text { Colombia, mediante la red social de twitter. }\end{array}$} \\
\hline Ocupación & & \\
\hline Prensa & & \\
\hline Privatización & & \\
\hline Sindicatos & & \\
\hline Afiliados & \multirow{5}{*}{ Salud } & \\
\hline Calidad & & \\
\hline Eficiencia/eficacia & & \\
\hline Politización & & \\
\hline Recursos & & \\
\hline Calidad & \multirow{6}{*}{ Educación } & \\
\hline Gratuidad & & \\
\hline Maestros & & \\
\hline Presupuestos & & \\
\hline Sociales & & \\
\hline Universalidad & & \\
\hline Amnistía e impunidad & \multirow{5}{*}{ Paz } & \\
\hline Cultivos de uso ilícito & & \\
\hline Institucional & & \\
\hline Líderes sociales & & \\
\hline Seguridad & & \\
\hline Social & \multirow{4}{*}{ Corrupción } & \\
\hline Lucha contra corrupción & & \\
\hline Institucional & & \\
\hline Ética & & \\
\hline
\end{tabular}

Tabla No 1 - Códigos abiertos, axiales y selectivo

Sergio Fajardo (SF), Candidato de la convergencia de partidos de Centro, movimiento "Compromiso ciudadano". Ex alcalde de Medellín y ex Gobernador de Antioquia (Página web oficial, campaña).

German Vargas (GV), es nieto del presidente de Colombia (1966 y 1970), candidato del partido oficialista, ex senador de la república, ex Ministro del Interior y Justicia. Fue vicepresidente de la república (2014 2017, durante el periodo de mandato del presidente Juan Manuel Santos (Página web oficial, campaña).

Ivan Duque (ID), Candidato de la coalición de partidos de Derecha, liderada por el ex presidente Álvaro Uribe, ex congresista en una oportunidad, es el candidato más joven de los cuatro que componen la muestra (Página web oficial, campaña). 


\section{Análisis de códigos y filiación política}

\subsection{Corrupción}

En relación con el código axial de Corrupción, el candidato $S F$ posee como línea fundamental de su campaña, un discurso de Lucha frontal contra la corrupción, tal y como lo expresa en el tweet del 25 de octubre, donde afirma que: "Es votando que derrotamos a los corruptos. La abstención es la mejor aliada de la corrupción. Votemos \#sepuede". Así, transmite mensajes en los cuales las personas poseedoras de redes sociales virtuales se sienten con la responsabilidad de generar luchas por la corrupción. Es de resaltar, la constancia del hashtag "Sepuede" en sus tweets, invitando a todos los ciudadanos a abordar una lucha en contra de algunos sectores políticos, que él y su equipo identifican como focos de corrupción.

Otro de los códigos abiertos utilizados para relacionar un tweet respecto al código axial de Corrupción por parte de $S F$, hace referencia a la Ética, el día 4 de octubre afirma que: "La corrupción es un problema ético. Con transparencia y sin comprar un voto \#Sepuede cambiar la política”. De esta forma, el candidato resalta sus valores éticos en pro de evitar la corrupción, destacando una diferencia con algunos candidatos presidenciales en la transparencia y compra de votos.

ID estructura sus tweets en relación al código Corrupción desde otra perspectiva. Para el candidato, la Corrupción desde la Institucionalidad, que tiene relación directa con el proceso de paz, el cual ha sido la bandera de un gobierno al que su corriente política se opone, muestra de esto es el tweet publicado el 21 de octubre, en el cual afirma: "Ojo con la otra corrupción, hacer conejo, engañar y moldear las leyes para blindar a los delincuentes es corrupción \#ForoCDSincelejo", es decir que el candidato otorga elementos de Corrupción al Congreso de la República.

También invita a la Lucha frontal contra la corrupción mediante el twitter, pues el día 14 de octubre, el candidato afirma que: "Le estamos proponiendo a Colombia, un país con una cultura de la legalidad. Gracias \#SantaRosaDeViterbo gracias \#Boyacá”, en el mensaje, puede observarse que establece diferencias entre él y otros candidatos presidenciales que a su juicio, no proponen cultura de legalidad. Otro de los códigos de resaltar del candidato ID es el componente Social, pues afirma que: "Para luchar contra la corrupción debemos inculcar valores desde la familia e inculcar cátedra de cívica en sistema educativo", se establece aquí, responsabilidades sobre la corrupción a otros agentes, se apela a la familia y las necesidades de cambios sociales, en otras palabras, el problema de la corrupción va más allá de la responsabilidad institucional.

El candidato GP, en relación al código axial Corrupción, propone principalmente una mirada desde el fortalecimiento de la Institucionalidad, apelando al papel de la participación ciudadana en la transformación de las estructuras corruptas y la lucha contra la Corrupción, proponiendo un nuevo presente de cambios. En el tweet publicado el 6 de octubre, afirma: "Mientras el Estado y la Justicia se hunden en corrupción y represión sanguinaria, la ciudadanía democrática se mueve, Bucaramanga ahora”.

Es pertinente resaltar que, durante el mes de octubre $G V$, no publica ningún tweet, en relación al código Corrupción. El candidato y su partido de pertenencia histórica ha sido 
uno de los más se han visto vinculados en casos judiciales y sentencias por Corrupción durante los últimos años.

El código abierto que tuvo mayor frecuencia de tweets por parte de los candidatos presidenciales, para este código axial, fue Institucional con un total de 32 tweets, 10 realizados por el candidato GP y 22 por el candidato $I D$, es importante mencionar que la alta repetición o referencia a este concepto de lo Institucional en la Corrupción, da muestra de la preocupación que se tiene en el país con respecto al nivel de confianza de la ciudadanía en las instituciones.

Por el contrario, el código abierto que registró menor frecuencia de tweets fue Ética con un total de 3 tweets, 2 para $I D$ y 1 para $S F$, lo que da muestra de que el tema de la Ética en la gestión pública poco a poco ha ido tomando importancia, que aún no es el tema principal de debate.

\subsection{Desempleo}

El candidato del Centro Democrático ID, analiza el desempleo desde varias perspectivas, es de resaltar la mención del emprendimiento y la Informalidad, expuesta en el tweet del 28 de octubre, en el cual afirma que: "Principio de emprendimiento incluye la lucha contra la informalidad: el mejor programa social en Colombia es un empleo formal \# DuquePropone”.

Por su parte, el candidato GP, hace alusión los medios de comunicación y la Ocupación de las clases menos favorecidas en ciudades como Bogotá. Mediante un tweet del 28 de octubre escribe: "Aún le dicen que paralizamos la ciudad de Bogotá? Lo engañan esta es la tasa de ocupación laboral entre 2005 y 2017”. Este Tweet, revela la defensa de su gestión como alcalde de la capital colombiana en un periodo determinado, llama a no creer en la información que han brindado sobre la misma los medios de comunicación, a los que tilda de mentirosos, por ello exhibe tasas favorables en su periodo.

El mismo candidato, muestra preocupación por los Sindicatos en el tema de Desempleo, pues en un tweet del 2 de octubre afirma que: "Todo el sindicalismo de las empresas públicas, ESP, del país y todos los recicladores deben alistarse a la arremetida que el fallo establece", puede observarse una posición crítica del candidato, frente a la facción política a la que se opone, la cual desmonta algunas medidas impulsadas por él en su periodo como alcalde, arremetiendo contra la privatización de empresas que se habían hecho públicas en su gestión. En otro tweet del mismo día, advierte con mayor puntualidad, que: "Fallo $2^{\mathrm{a}}$ instancia de Tribunal de Cund contra Acueducto, destruye todas las empresas creadas por empresas públicas ESP”, el candidato alude a la privatización como uno de los principales causantes del Desempleo. Durante el mes de octubre, $S F$ y $G V$, no publican ningún tweet, en relación al desempleo.

Para el código axial Desempleo, los códigos abiertos que registraron mayor frecuencia de tweets fueron Informalidad y Ocupación, 12 para Informalidad (12 tweets de ID) y 12 para Ocupación (3 de GP y 9 de ID), ambos temas generaron una alta emisión de tweets por parte de los candidatos, lo que da muestra de la constante preocupación que se tiene respecto al Desempleo.

El código abierto que registró menor frecuencia de tweets fue Sindicatos, con solo 1 tweet emitido por el candidato GP. 


\subsection{Educación}

La Educación, por su parte, ha sido uno de los temas abordados por todos los candidatos presidenciales en la red social twitter. El candidato $S F$, reconocido como una figura en pro de los procesos educativos, se caracterizó por tweets dedicados a la generación de cultura, ligándolo al código abierto Sociales, pues el 14 de octubre, afirma que: "El mayor privilegio en la vida: hacer lo que nos apasiona. \#sepuede vivir para leer: la revolución de la cultura".

El candidato, GP, se encamina por propuestas que conlleven al aumento de Presupuesto que permita la Universalidad en la educación, mediante la Gratuidad de la misma, es decir 3 elementos discursivos en una publicación; muestra de esto, es el tweet publicado el día 4 de octubre: "me propongo a lograr que juventud pueda acceder sin exclusión a la universidad pública y gratuita". El 19 de octubre, alude la importancia de contar con los educadores (Maestros - código abierto) en su propuesta, convirtiendo a estos en actores claves de su programa de gobierno, aquí expresa: "En diálogo con los maestros y maestras de Antioquia sobre la política educativa de la Colombia Humana".

El día 22 de octubre del año, comenta: "1er requisito de la III revolución industrial en Colombia: una educación pública, de calidad, universal y financiada como una prioridad", se revelan cuatro elementos discursivos: Calidad de la Educación, Gratuidad, Presupuesto y Universalidad de la educación. Por último, alude a la necesidad de un nuevo enfoque de la Educación, que se preocupe por la relación entre lo que enseña el sistema educativo y las necesidades del país, el día 22 de octubre, expresa que: "Educación pública que necesita Colombia debe generar amor a la naturaleza, a la colaboración y no a la competencia”.

$G V$ se preocupa por aspectos Sociales ligados a la Educación, pero desde una perspectiva diferente a la propuesta de GP, pues en un tweet del 21 de octubre manifiesta: "En poco días presentaré mis propuestas de gno. Educación tiene gran énfasis. Jóvenes deben ser educados en emprendimiento y competitividad", es decir la competitividad como principio de la Educación, diferente a lo propuesto por GP, el cual le quiere restar incidencia a este aspecto.

$I D$, aborda el tema desde distintas perspectivas, una de ellas es la Calidad de la Educación, enfocándola con el tipo de alimentación brindado a los niños, pues el día 28 de octubre comenta: “(...) la educación en Colombia requiere una política integral que comienza garantizando nutrición de más vulnerables, con hambre no se aprende". La Gratuidad y el Presupuesto se convierten en ejes de su propuesta educativa, el 24 de octubre afirma que: "Hay que buscar que las universidades públicas no estén desfinanciadas, ampliar gratuidad e incentivar la educación virtual”.

En referencia al código axial Educación, el código abierto que registró mayor frecuencia de tweets fue Calidad, con un total de 30 tweets, 1 para GP, 3 para GV y 26 para $I D$.

Por otra parte, el código abierto que registró menor frecuencia en este código axial fue Maestros, pues solo se registraron 3 tweets, 1 emitido por GP y 2 emitidos por ID.

\subsection{Salud}

$G V$ presenta una única publicación realizada en relación al tema, se preocupó por la Calidad de la misma, aunque su principal objetivo era brindar un panorama general sobre sus propuestas, el 22 de octubre escribe: "Colombia entera sabe que, para tener mejor educación, más y mejor viviendas y vías, mejor salud, mayor seguridad... \#MejorVargasLleras”. Se une 
a la preocupación por la Educación con áreas en las que trabajó como ministro durante el gobierno vigente del presidente Juan Manuel Santos, en temas como vivienda y vías y de seguridad en el que trabajo durante el gobierno de Álvaro Uribe.

$G P$, pone de presente su preocupación por el tema de los Recursos asignados para el sistema de salud colombiano, criticando así, posturas propuestas por el candidato presidencial $G V$ y el alcalde de Bogotá, Enrique Peñalosa, mediante un tweet el día 11 de octubre, comenta: "Vargas Lleras y Peñalosa, cómo se les ocurre quitar incentivo de Sisben, subir pasajes y esperar quiebre transporte en Ciudad Bolívar", se apela a la defensa de los subsidios y ayudas para lograr el acceso de las clases menos favorecidas al sistema. Este discurso se apoya en otras publicaciones como la del 10 de octubre: "Derrumbe del sistema de salud/Bogotá: caen servicios ofrecidos por hospitales públicos, sube cartera, caen recaudos, se dispara déficit”.

$I D$, se encarga de abordar el tema de la Salud de diferentes perspectivas, precisa que el número de Afiliados en la Salud no debe ser un indicador de la misma, lo que sí debe ser la Calidad, en el tweet publicado el día 24 de octubre, expresa: “A las EPS tenemos que dejar de remunerarlas solo por el número de afiliados, tenemos que evaluarla por la calidad de servicio”. La Politización de la misma, es otro de los temas abordados por el candidato del Centro Democrático, pues el 10 de octubre afirma que: “\#8IdeasParaLaSalud: 3. Para que funcione el sistema de salud tenemos que poner freno a corrupción y acabar con abusos de contratación directa”. Por último, un aspecto que no fue tratado por los demás candidatos presidenciales en lo referente a la Salud fue la Eficiencia y eficacia, pues el día 10 de octubre, ID, expresó: “\#8IdeasParaLaSalud: 6. Necesitamos una política de trasplantes que contribuya a un sistema de salud más efectivo y eficiente”. En relación a la Salud, durante el mes de octubre, SF no publica ningún tweet.

Es menester mencionar que en este código axial se registraron la menor cantidad de tweets (22), en ese sentido, el código abierto que tuvo mayor frecuencia de tweets para este código fue Recursos, con un total de 9 tweets, 4 para GP y 5 para ID.

Por el contrario, los códigos abiertos que registraron menor cantidad de tweets para este código axial fueron Eficiencia/Eficacia, Afiliados y Politización, con un total de 6 tweets para los 3 códigos (todos emitidos por ID), 2 tweets para Eficiencia/Eficacia, 2 para Afiliados y 2 para Politización.

\subsection{Paz}

Teniendo en cuenta el marco de posconflicto por el cual pasa Colombia, el código axial $P a z$, es el más abordado por los cuatro candidatos presidenciales. Por una parte, $S F$ y GP poseen discursos ligados a superar la etapa del conflicto armado en Colombia, sin embargo, $G V$ e $I D$ exhiben discursos críticos frente al proceso de paz realizado en La Habana con las FARC-EP, proponiendo así reformas y/o alternativas.

De este modo, $S F$ se caracteriza por brindar un aspecto Institucional de la paz, en la cual las instituciones deben convertirse en garantes, el tweet publicado el día 9 de octubre afirma: "Tumaco: el reto de Colombia. El primer lugar donde tenemos que mostrar que significa construir la paz \#sepuede. No más destrucción y dolor”, aludiendo a la masacre de líderes efectuada día antes y expresando la necesidad de fortalecer aspectos institucionales, mediante la presencia Estatal. 
$G P$, involucra la preocupación generalizada de los partidos y movimientos de izquierda por los Líderes sociales en Colombia, el 29 de octubre escribe: "El ELN se sumó a los victimarios de líderes sociales en Colombia. Aulio Isarama, su víctima es pueblo Colombiano". Sin embargo, el candidato también resalta la necesidad de un Estado que cumpla los acuerdos de paz realizados en La Habana, es decir desde la Institucionalidad, pues el 25 de octubre reclama que: "El dato real no es que las Farc se tomó el país, sino que el Estado le puso conejo al acuerdo con las Farc".

La generación de Seguridad a partir de la construcción de Paz, también es un elemento importante para el candidato GP, el 18 de octubre asevera que: "En Somalia van 315 muertos por un atentado con camión bomba, eso le espera a las sociedades que no son capaces de hacer paz". La sustitución voluntaria de Cultivos, es otro tema tratado por el candidato, pues esta plantea como la mejor opción frente al problema de los Cultivos ilícitos, sin embargo, este ejercicio se ha visto afectado por la muerte de Líderes sociales, configurando dos tipologías discursivas, así el 9 de octubre, plantea: "Quienes asesinan a campesinos cultivadores de coca desde el Estado, buscan sabotear la opción de la sustitución voluntaria de cultivos".

Por su parte, $G V$, asigna un rol fundamental a las Instituciones en la construcción de $P a z$, el 16 de octubre dice: "Desmovilización y entrega de las Farc fueron valiosas. Q todo salga bien depende d adecuada implementación. La LeyEstatutaria es fundamental”. Sin embargo su preocupación por la Amnistía e impunidad es expresada en algunos tweets, pues el día 16 de octubre se pregunta: “¿Cómo entender que Ley Estatutaria habilite a participar en política a quienes NO han concurrido a la JEP?”, denotando rechazos a los acuerdos realizados en La Habana. Otra de la muestras de esto, es la relación de Cultivos de uso ilícito con zonas veredales, pues el 16 de octubre expresa: "Insisto, miren como coinciden zonas veredales con cultivos de coca", a lo cual anexa un mapa de Colombia que permite correlacionar los datos de zonas veredales (Lugares de concentración de los antiguos combatientes de las FARC) y hectáreas de Cultivos ilícitos.

Finalmente, $I D$, puede considerarse el más contradictorio al proceso de paz, el 27 de octubre, publica: "El gobierno Santos sigue creando incentivos para sembrar coca 5.2 canchas como el Campín lo puede hacer", es decir que programas de sustitución, que otorguen incentivos económicos son muy criticados por el candidato. Estar al tanto de las situaciones del acuerdo, para expresar Amnistía e impunidad se convierte en otra tendencia del candidato, el 27 de octubre se cuestiona: “¿Qué está esperando el Ministerio de Defensa para decirle al país como van a capturar a este sujeto? Ahora van a decir que es un disidente". Respecto a la Seguridad, el candidato considera a la disidencia un gran peligro, pues expresa que: "Farc negociaron como organización monolítica y de mando unificado y hoy disidencias amenazan seguridad nacional”. Es de resaltar la presencia de hashtags en el perfil del candidato como: \#noalaimpunidad \#reformarlajep.

Con un total de 72 tweets, 22 para $G V$ y 50 tweets para $I D$, el código abierto de mayor frecuencia para el código axial Paz, fue Amnistía e impunidad, siendo el código que registró más cantidad de tweets de todos los códigos que se identificaron, y es que el tema de la Amnistía y la impunidad se convirtieron en conceptos muy abordados. Por el contrario, un tema de poca recurrencia y abordaje por parte de los candidatos presidenciales durante el mes de análisis fue el código abierto Líderes sociales, con un total de 7 tweets, 3 para GP y 4 para $I D$. 


\section{Conclusión}

Las redes sociales virtuales han constituido nuevas dinámicas sociales y políticas de la realidad, estableciendo particularidades de estudio para las ciencias sociales y las democracias contemporáneas. De este modo, los candidatos presidenciales, optan por el frecuente uso de sus redes sociales virtuales con el fin de aumentar su potencial electoral.

Candidatos presidenciales, los cuales expresan poca o ninguna preocupación por algunas temáticas, da muestra de orientaciones políticas definidas. La nula interacción del candidato Germán Vargas Lleras por el tema de la Corrupción, dista de lo expuesto por GV y SF, los cuales invitan a derrotar la Corrupción en las urnas, estableciendo diferencias discursivas. El tema de la Paz, significó posiciones discursivas distanciadas, por una parte GV e ID, adoptan posiciones discursivas referentes a la Amnistía e impunidad brindada a los miembros de las FARC-EP en los acuerdos de paz. Por su parte, SF y GP, propician espacios de Paz desde la Institucionalidad, obedeciendo a pasados y trayectorias políticas particulares.

Las posiciones discursivas de los candidatos presidenciales en Colombia, responden a dimensiones políticas, pues la filiación de estos a movimientos, grupos, organizaciones y/o partidos políticos con tendencias ideológicas definidas, opta por configurar temáticas a abordar, teniendo como referentes las principales percepciones de los ciudadanos, realizando incorporaciones de problemáticas desde la agenda sistémica a la agenda pública y de medios. De este modo, el microanálisis del discurso, a partir de la configuración de 25 categorías, producto de 5 temas establecidos (Paz, Educación, Salud, Corrupción y Desempleo) con el fin de colegir en un único código medular, permite establecer comparaciones entre los candidatos respecto a temas. La metodología empleada, permite la generación de teoría fundamentada, a partir de la revisión línea a línea de los tweets emitidos por los candidatos en el mes de octubre del año 2017.

\section{Referencias}

Alcaldía de Popayán. (2014). Plan Integral único 2011-2015. Alcaldía de Popayán.

Ancona, M.Á. (1995). Metodología cuantitativa: estrategias y técnicas de investigación social. Barcelona: Editorial Síntesis.

Arendt, H. (1997). ¿Qué es la política?. Barcelona: Paidós.

Castoriadis, C. (2007). La institución imaginaria de la sociedad. Buenos Aires: Tousquest.

Duque, I. (2018). Página web oficial, campaña. (S.F). Duque. Recuperado de: https://www.ivanduque.com/

Durkheim, E. (1997). Las reglas del método sociológico. México: Fondo de Cultura Económica.

Echeverría, B. (2011). Lo político en la política. En: Bolivar Echeverría. Ensayos políticos. Quito: Pensamiento político ecuatoriano. 
Fajardo, S. (2018). Página web oficial, campaña. (S.F). Fajardo presidente. Recuperado de: http://sergiofajardo.co/

Gutiérrez, F. C. (2009). Análisis sociológico del sistema de discursos. Madrid: Centro de investigaciones sociólogicas.

Mayorga, F., \& Córdova, E. (2007). Gobernabilidad y Gobernanza en América Latina. Gobernabilidad Y Gobernanza En: América Latina - Working Paper NCCR NorteSur IP8, Ginebra., pp. 18-20.

Mejor Vargas Lleras (s.d.) Página web oficial, campaña. (S.F). Mejor Vargas Lleras. Recuperado de: https://www.mejorvargaslleras.com/?gclid=EAIaIQobChMIoICV 7urf2gIVAh-GCh2UzAiVEAAYASAAEgIuDvD_BwE

Moreno Montero, F. (2017). Use of virtual social networks and citizen participation in Podemos. Teknokultura, 14(2), 351-362. http://dxd.oi.org/10.5209/TEKN.56099.

Mouffe, C. (2011). En torno a lo político. Buenos Aires: Fondo de Cultura Económica.

Outeriño, B. (2014). Conectados por redes sociales: Introducción al Análisis de redes sociales y casos prácticos. Redes, Revista Hispana Para El Análisis de Redes Sociales, 26(2), 236-241. Recuperado de: https://doi.org/10.5565/rev/redes.548

Ruano, L., Congote, E., \& Torres, A. (2016). Comunicación e interacción por el uso de dispositivos tecnológicos y redes sociales virtuales en estudiantes universitarios. RISTI - Revista Iberica de Sistemas e Tecnologias de Informacao, (19), 15-31.

Ruano, L. E., López, J. C., \& Mosquera, J. F. (2018). Dimensión política del discurso en los candidatos presidenciales de Colombia: un análisis de la red social Twitter. En: Atas do $7^{\circ}$ Congresso Ibero-Americano em Investigaçao Qualitativa. Investigação Qualitativa na Educação. Vol. 3, pp. 301-310 Fortaleza, Brasil: Ludomedia.

Retamozo, M. (2010). Lo político y la política. Sujetos políticos, conformación y disputa por el orden social. Revista Mexicana de Ciencias Políticas y Sociales, (206).

Sant, D., Ramos, A., Pocrifka, D. H., \& Simonian, M. (2016). Associação entre a técnica de análise de conteúdo e os ciclos de codificação: possibilidades a partir do software ATLAS. ti. RISTI - Revista Iberica de Sistemas e Tecnologias de Informacao, (19), 93-106.

Schmitt, C. (2009). El concepto de lo político. Madrid: Alianza Editorial.

Semana, R. R. (2016). Revista Semana. Recuperado de: https://www.semana.com/ educacion/articulo/crisis-de-la-profesion-docente/473152. Acedido en 20 de 10 de 2018.

Semana, R. R. (2017). Revista Semana. Recuperado de: https://www.semana.com/ educacion/articulo/informe-unesco-sobre-educacion-en-america-latina/542592. Acedido en 20 de 10 de 2018.

Semana, R. R. (2018). Revista Semana. Recuperado de: https://www.semana. $\mathrm{com} /$ nacion/articulo/lideres-asesinados-en-colombia-en-debate-en-camaradenuncian-exterminio/582020. Acedido en: 20 de 10 de 2018. 
Strauss, A., \& Corbin, J. (2002). Bases de la investigación cualitativa: técnicas y procedimientos para desarrollar la teoría fundamentada. Recuperado de: https://doi.org/10.4135/9781452230153

Turpo, O. (2008). La netnografía: un método de investigación en Internet. Educar, 42, 81-93. Recuperado de: https://doi.org/10.5565/rev/educar.134

Villanueva, L. A. (1993). Problemas públicos y agenda de gobierno. México: Miguel Ang. 